\title{
Contralesional homotopic functional plasticity in patients with temporal glioma
}

\author{
*Dongming Liu, MS,1 Jiu Chen, MD,, 2,3 Xinhua Hu, MD,, ${ }^{1,3}$ Guanjie Hu, MS, ${ }^{1}$ Yong Liu, MD,1 \\ Kun Yang, MD, ${ }^{1}$ Chaoyong Xiao, MS, ${ }^{3,4}$ Yuanjie Zou, MS, ${ }^{1,3}$ and Hongyi Liu, MD ${ }^{1,3}$

\begin{abstract}
'Department of Neurosurgery, The Affiliated Brain Hospital of Nanjing Medical University, Nanjing, Jiangsu; ${ }^{2}$ Institute of Neuropsychiatry, The Affiliated Brain Hospital of Nanjing Medical University, Fourth Clinical College of Nanjing Medical University, Nanjing, Jiangsu; ${ }^{3}$ nstitute of Brain Functional Imaging, Nanjing Medical University, Nanjing, Jiangsu; and ${ }^{4}$ Department of Radiology, The Affiliated Brain Hospital of Nanjing Medical University, Nanjing, Jiangsu, China
\end{abstract}

\begin{abstract}
OBJECTIVE This study aimed to explore the contralesional homotopic functional plasticity in the brain of patients with unilateral temporal glioma.

METHODS Demographic, neurocognitive, and resting-state functional MRI data were collected from 17 patients with temporal glioma (10 in the right lobe and 7 in the left lobe), along with 14 age- and sex-matched healthy controls. The amplitude of low-frequency fluctuation (ALFF) of the contralesional homotopic region and 2 control regions was examined. The region-of-interest-based analysis was used to determine the altered functional connectivity (FC) of the contralesional homotopic region, showing significantly different intrinsic regional brain activity between patients and controls. Partial correlation analysis was conducted to determine the association between the altered neural activity and behavioral characteristics.
\end{abstract}

RESULTS Compared with controls, patients with right temporal glioma exhibited significantly increased ALFF in the contralesional homotopic hippocampus and parahippocampal region. In addition, the intrinsic regional activity in these regions was negatively correlated with the visuospatial score $(r=-0.718, p=0.045)$. Whole-brain FC analysis revealed significantly increased FC between the left hippocampus and parahippocampal regions and the left inferior temporal gyrus, and decreased FC between the left hippocampus and parahippocampal regions and the left inferior frontal gyrus. No significant changes were found in the 2 control regions.

CONCLUSIONS Contralesional homotopic regions are instrumental in the process of neural plasticity and functional compensation observed in patients with unilateral temporal glioma. The observed findings might be used to help preoperative evaluation or rehabilitation of postsurgical patients.

https://thejns.org/doi/abs/10.3171/2019.11.JNS191982

KEYWORDS resting-state MRI; cognitive function; temporal glioma; plasticity; contralesional hippocampus; oncology

$\mathrm{G}$ LIOMAS account for approximately $81 \%$ of malignant brain neoplasms and are the most common primary intracranial tumors. ${ }^{26}$ In addition to common neurological manifestations, multiple (mild) cognitive deficits are also frequently observed in glioma patients. ${ }^{30}$ Previous studies have indicated that patients with glioma might experience potential neural adaptation to the lesions. ${ }^{1,13,43}$ Faced with a progressive loss of neural tissue, the brain may reassign the remaining physiological resources to maintain a compensatory level of function in a socially and cognitively demanding environment. ${ }^{14}$ An increasing number of studies have identified different patterns of lesion-induced cerebral plasticity by functional imaging, ${ }^{6,714,17,33}$ which are related to the location, extent, type, and development of the lesions. Brain plasticity has been observed in the perilesional, ipsilateral hemispheric, contralateral hemispheric, and cerebellar regions ${ }^{1,13,43}$ and manifests as structural rearrangement and/or functional compensation., ${ }^{1,16,38}$ Functional compensation can be classified into specific function and functional networks. ${ }^{13,16,20}$

Functional compensation frequently manifests as altered blood flow or metabolism at the resting state or

ABBREVIATIONS ALFF = amplitude of low-frequency fluctuation; DSST = Digital Symbol Substitution Test; FC = functional connectivity; fMRI = functional MRI; GRF = gaussian random field; HGG = high-grade glioma; $L G G$ = low-grade glioma; $\mathrm{MNI}=$ Montreal Neurological Institute; $\mathrm{ROI}$ = region of interest; TFCE-FWE = threshold-free cluster enhancement family-wise error.

SUBMITTED July 22, 2019. ACCEPTED November 5, 2019.

INCLUDE WHEN CITING Published online January 10, 2020; DOI: 10.3171/2019.11.JNS191982.

${ }^{*}$ D.L., J.C., and X.H. contributed equally to this work and share first authorship. 
during activation and can be tracked via functional imaging to study brain recovery and plasticity in a noninvasive manner. ${ }^{13}$ Functional MRI (fMRI) has been used to explore the neural plasticity in patients with glioma. ${ }^{11,43}$ The amplitude of spontaneous brain oscillations can be measured as the amplitude of low-frequency fluctuations (ALFFs) to investigate the disturbances in the resting-state intrinsic regional activity. ALFF is a feasible and reliable indicator of resting-state regional brain activity, ${ }^{41}$ which is altered in the presence of brain lesions. ${ }^{12,43}$ ALFF has been used to measure diverse brain plasticity, including the sensorimotor system, ${ }^{23}$ motor recovery, ${ }^{21}$ reorganization of cerebro-cerebellar circuits, ${ }^{43}$ and functional networks. ${ }^{19}$ Altogether, this suggests that ALFF is a reliable tool to investigate brain disorders and the concomitant functional plasticity.

Previous studies have shown that slow but massive infiltration of the insula with glioma masses can induce a marked increase in gray matter volume in the contralateral insula. The homotopic reorganization in the macrostructure of the cortex might be a physiological basis of functional compensation. ${ }^{1}$ In addition, empirical studies have demonstrated the importance of contralateral homotopic areas in maintaining cognitive and sensorimotor functions. ${ }^{10,37}$ Despite the critical role of the temporal structure in multiple brain functions, ${ }^{22}$ surgical excision of the tumor-infiltrated temporal lobe may not result in serious neuropsychological impairments ${ }^{14}$ underscoring functional compensation and neural plasticity in patients with unilateral focal glioma. Furthermore, unilateral or focal glioma growth not only remodels the regional neural activity but also alters the large-scale neural network. $4,24,43$ Reorganization of the resting-state networks has been observed in various pathological conditions involving brain damage, such as stroke and brain tumors. ${ }^{5,20,36}$ It is, however, not completely clear whether the functional reorganization in patients with hemispheric focal glioma is supported by modifications in the contralesional homotopic region, or if that plays any role in global network integration.

To this end, we used ALFF to explore the functional remodeling in patients with temporal glioma and selected possible regions for subsequent region-of-interest (ROI)based functional connectivity (FC) analysis. We hypothesized that the homotopic temporal lobe supports functional compensation, which can be detected by ALFF and might reflect the alteration (or at least a trend of the alteration) of clinical manifestations in some way. We further hypothesized that the homotopic functional compensation is not isolated and involves the entire neural network of the brain.

\section{Methods \\ Participants}

We reviewed the records of 32 patients who underwent temporal/insular tumor surgery at the Department of Neurosurgery, The Affiliated Brain Hospital of Nanjing Medical University, Jiangsu Province, China. Since the tempo$\mathrm{ral} /$ insular glioma localization is frequent, ${ }^{6,27}$ the clinical and MRI data of these patients were readily available. The inclusion criteria were as follows: 1) histopathologically confirmed primary glioma (tumor grade was reexamined by the pathologist according to the 2016 WHO classification of central nervous system tumors); 2) no evidence of shift of the midline structures (septum pellucidum, corpus callosum, third ventricle) due to peritumoral edema or mass effect of the lesion as confirmed by structural images; 3) no history of head trauma, cerebrovascular disease, psychological disease, temozolomide chemotherapy, or radiotherapy; and 4) absent or slight neurological focal deficit including aphasia or paresis. The exclusion criteria were as follows: 1) recurrent glioma; 2) multiple lesion foci; 3) history of substance abuse, including tobacco and alcohol; and 4) inadequate MRI data acquisition and preprocessing. Based on these criteria, 17 patients (mean age $53.59 \pm 13.13$ years, 11 males and 6 females) were included in the study. In addition, 14 age- and sex-matched healthy controls were recruited from the local community, after excluding those with a history of head trauma, severe systemic disease, or psychological disorders. All participants provided written informed consent, and the study was approved by the Institutional Ethical Committee for Clinical Research of the Affiliated Brain Hospital of Nanjing Medical University.

\section{Neurocognitive Assessment}

All patients were evaluated during a standardized clinical interview and neurocognitive tests. The details of the neurocognitive assessment are provided in Supplemental Data Methods S.2.3.

\section{MRI Data Acquisition}

Patients underwent preoperative MRI between 2013 and 2015. All MRI images were acquired on a 3.0-T Verio scanner (Siemens) at the Department of Radiology, the Affiliated Brain Hospital of Nanjing Medical University. The details of image acquisition parameters are provided in Supplemental Data Methods S.2.3 and in our previously published study. ${ }^{20}$

\section{Lesion Drawing and Mask Fabricating}

Original T1-weighted images were normalized to the Montreal Neurological Institute (MNI) Template using SPM12 (https://www.fil.ion.ucl.ac.uk/spm/software/ spm12/) with a spatial resolution of $2 \times 2 \times 2 \mathrm{~mm}$. The outline of the lesion was then traced manually on individual 3D T1-weighted images using MRIcron software (https:// www.mccauslandcenter.sc.edu/crnl/mricron/). The details of image acquisition parameters are provided in Supplemental Data Methods S.2.4.

\section{MRI Imaging Data Preprocessing}

MRI images were preprocessed using Data Processing and Analysis for Brain Imaging (DPABI; http://www. rfmri.org) toolkit ${ }^{39}$ and Statistical Parametric Mapping 12 (SPM12; http://www.fil.ion.ucl.ac.uk/spm) toolkit in MATLAB (release 2013b, http://www.mathworks.com/ products/matlab/). The details of imaging data preprocessing are provided in Supplemental Data Methods S.2.5. 


\section{Contralesional Intrinsic Spontaneous Neural Activity Analysis}

ALFF was used to investigate the intrinsic spontaneous neural activity of the contralesional homotopic region at the voxel level since it can reflect changes of neural activity effectively on fMRI. ${ }^{9,12}$ The details of ALFF calculation are provided in Supplemental Data Methods S.2.6. The statistical analysis of ALFF was confined to the homotopic masks of the contralesional hemisphere.

To verify that the functional compensation was specific to the contralesional homotopic temporal lobe, the ALFF of the temporal lobe and 2 control regions (thalamus and medial frontal) were also calculated. ${ }^{1}$ The reason for choosing these regions in the contralesional hemisphere is that the thalamus near the midline might be sensitive to tumor mass effect (midline shift), while the medial frontal region is relatively far from the contralesional homotopic regions. To assess the effects of the tumor grade on fMRI changes, we also performed analysis ignoring side but comparing contralateral neural activity in low-grade versus high-grade tumors. The ALFF values of the patients and controls were compared using t-tests, and the results were corrected by the threshold-free cluster enhancement family-wise error (TFCE-FWE) correction. ${ }^{34}$ The standardized ALFF values of the regions showing significant group differences and that of the control regions were also extracted and compared with that of the healthy controls.

\section{ROI-Based Whole-Brain FC Analysis}

To study the reorganizational pattern of the homotopic region from a network perspective, the regions within the homotopic mask showing significant group differences in ALFF were defined as ROIs for the subsequent FC analysis. ${ }^{43}$ The cluster saved was corrected by TFCE-FWE correction $(p<0.05)$. The signal time courses of the ROI were extracted using the Resting-State fMRI Data Analysis Toolkit (REST, http://resting-fmri.sourceforge.net) software, and voxel-level correlation analysis was performed to generate the FC map in each subject. The whole-brain FC map was calculated using the Functional Connectivity Toolkit in REST. The resultant FC maps were transformed into $\mathrm{z}$ scores by Fisher's r-to-z transformation to improve normality for the subsequent t-test. Statistical analysis of the FC map z scores was confined to the nonlesional whole-brain gray matter mask.

\section{Statistical Analysis}

Statistical analysis was performed using IBM SPSS (version 19.0, IBM Corp.). An independent two-sample ttest was used to compare the demographic variables and neurocognitive scores. The significance threshold was set to a Bonferroni corrected $\mathrm{p}$ value $<0.05$ ( 7 tests). The sex ratio was compared using the chi-square test, and nonparametric permutation tests were used to compare the ALFF values between patients and controls ${ }^{34}$ using the DPABI toolkit. ${ }^{3}$ The TFCE-FWE-corrected cluster $p$ value $<0.05$ was considered statistically significant. The permutation times were set at 1000 tests. Gaussian random field (GRF) correction was used for FC analysis, and voxel-level p $<0.0005$ and corrected cluster-level $\mathrm{p}<0.05$ were con- sidered statistically significant. Age, sex, and education (years) were treated as covariates in all between-group statistical analyses. Pearson's partial correlation analysis was conducted to analyze any association between the ALFF or FC values and clinical scores. Any p value < 0.05 was considered statistically significant.

\section{Results \\ Demographic and Clinical Characteristics}

The demographic data and clinical characteristics collected from all participants are presented in Table 1 and Table S1. All patients and controls were of Chinese Han descent and right-handed according to the Edinburgh Handedness Inventory. In our study, the period between the patient's first onset of subjective symptoms and the magnitude of resting-state changes did not exceed 24 weeks (all patients: mean 7.76 weeks [SD 7.79 weeks]). Patients with low-grade glioma (LGG) in the right temporal lobe tended to have a longer interval than those with highgrade glioma (HGG) in the left temporal lobe before the collection of fMRI data $(\mathrm{p}=0.047, \mathrm{t}=2.40)$. According to WHO classification, 8 patients had LGGs (WHO grade I or II) and 9 had HGGs (WHO grade III or IV). In addition, 7 gliomas were localized to the left temporal lobe (1 included the insula, $\mathrm{LGG} / \mathrm{HGG}=1 / 6$ ) and 10 to the right temporal lobe ( 2 included the insula, $\mathrm{LGG} / \mathrm{HGG}=7 / 3$ ). No significant differences were observed in age, sex, or education of the patients and controls. When evaluating neurocognitive characteristics, Bonferroni correction adjustments showed that all patients with temporal glioma exhibited inferior visuospatial, Digital Symbol Substitution Test (DSST), mapping, similarity, mathematics, and memory performances (all $\mathrm{p}<0.05$ ). Patients with right temporal lobe gliomas especially demonstrated worse performance in visuospatial, DSST, mapping, and mathematics tests (all $\mathrm{p}<0.05$ ), but not in memory and similarity tests $(\mathrm{p}>0.05)$.

\section{ALFF Group Differences in the Contralateral Homotopic Region}

In patients with right temporal lobe glioma $(n=10)$, the maximum lesion overlap was located within the right temporal lobe (Fig. 1A). Compared with controls, these patients showed increased ALFF in the left hippocampus and parahippocampal gyrus within the homotopic mask (permutation test, TFCE-FWE corrected $\mathrm{p}<0.05$ ). Details of the cluster are listed in Table 2. In patients with left temporal glioma $(n=7)$, the maximum lesion overlap was located within the left temporal and insular lobes (Supplemental Data Fig. S1A). No significant difference in ALFF analysis was found between these patients and controls. For the other control regions (i.e., the contralesional thalamus ROI and medial frontal ROI), the ALFF values were similar for both patient groups and controls. Compared with controls, the ALFF values extracted from the left hippocampus and parahippocampal gyrus were superior in patients with right temporal lobe glioma for the temporal lobe $(\mathrm{t}=5.12, \mathrm{p}<0.001)$, but not for the contralesional thalamus $(\mathrm{t}=0.45, \mathrm{p}=0.65)$ and the thalamus $(t=1.16, p=0.26$; Fig. 1D), confirming that the intrinsic 
TABLE 1. Demographics and cognitive measures of patients with temporal glioma and controls

\begin{tabular}{|c|c|c|c|c|c|c|c|}
\hline Items & HCs $(n=14)$ & All Patients $(n=17)$ & TempR $(n=10)$ & $\mathrm{t}\left(\chi^{2}\right)$ Value $^{*}$ & p Value* & $\mathrm{t}\left(\chi^{2}\right)$ Value $\dagger$ & p Value \\
\hline Age, yrs & $48.57(8.65)$ & $53.59(13.13)$ & $50.30(15.50)$ & -1.225 & 0.230 & -0.319 & 0.755 \\
\hline Sex, male/female & $5 / 9$ & $11 / 6$ & $6 / 4$ & 2.584 & 0.108 & 1.386 & 0.239 \\
\hline Education level, yrs & $9.50(4.83)$ & $7.94(3.17)$ & $9.00(2.50)$ & 1.079 & 0.289 & 0.300 & 0.767 \\
\hline Handedness, It/rt/ambił & $0 / 14 / 0$ & $0 / 17 / 0$ & $0 / 10 / 0$ & NA & NA & NA & NA \\
\hline \multicolumn{8}{|c|}{ Scores of each cognitive domain } \\
\hline DST & $11.00(2.26)$ & $8.09(2.88)$ & $8.38(3.11)$ & 2.367 & 0.210 & 1.927 & 0.518 \\
\hline Memory test & $11.88(1.55)$ & $4.27(4.76)$ & $5.63(4.93)$ & 4.951 & $0.001 \S$ & 3.423 & 0.059 \\
\hline Visuospatial test & $10.63(1.60)$ & $4.82(4.40)$ & $4.13(3.68)$ & 4.027 & $0.001 \S$ & 4.581 & $0.003 \S$ \\
\hline DSST & 11.88 (1.64) & $4.09(4.32)$ & $4.13(4.70)$ & 5.455 & $0.001 \S$ & 4.400 & $0.013 \S$ \\
\hline Mapping & $9.88(0.64)$ & $4.82(2.79)$ & $4.50(2.88)$ & 5.812 & $0.001 \S$ & 5.155 & $0.007 \S$ \\
\hline Similarity & 10.00 (1.07) & $5.00(3.52)$ & $5.63(3.70)$ & 4.437 & $0.005 \S$ & 3.212 & 0.084 \\
\hline Math exam & 10.63 (1.99) & $5.82(2.44)$ & $6.50(2.33)$ & 4.559 & $0.001 \S$ & 3.803 & $0.013 \S$ \\
\hline
\end{tabular}

Ambi = ambidextrous; DST = Digit Span Test; $\mathrm{HC}=$ healthy control; NA = not applicable; TempR = patients with right temporal glioma.

Values are expressed as the mean (SD) unless indicated otherwise. All p values were obtained using the t-test except for sex (chi-square test). Comparisons between controls and patients with left temporal glioma were not performed because 4 patients did not complete all cognitive tests.

* Two-sample t-tests between all patients with temporal glioma and controls.

† Two-sample t-test between patients with right temporal glioma and controls.

$\ddagger$ Values are number of patients.

$\S$ Significant differences were found between controls and patients with glioma. Bonferroni correction for multiple comparisons was performed at a significance level of $p<0.05$ (7 tests).

regional activity compensation was specific to the contralateral homotopic region. When we ignored the side but compared contralateral ALFF changes in low-grade versus high-grade tumors, patients with LGG $(n=7)$ in the right temporal lobe exhibited similar hyperactivity in contralateral homotopic regions, which did not present in patients with left temporal lobe HGGs $(n=6)$ (Supplemental Data Figs. S2-S4 and Table S2).

\section{Reorganization of Global FC in Patients With Right Temporal Lobe Glioma}

In patients with right temporal lobe glioma, the increased ALFF in the left hippocampus and parahippocampal gyrus (Fig. 1B) was selected as the ROI. Compared with controls, this group exhibited significantly increased FC to the left inferior temporal gyrus (voxel $p$ $<0.0005$, GRF corrected cluster $p<0.05$ ). In addition, this group showed significantly decreased FC to the left inferior frontal gyrus (voxel $\mathrm{p}<0.0005$, GRF corrected cluster $p<0.05)$. The details of the regions are summarized in Table 2.

\section{Correlation Between the Altered Intrinsic Regional Activity and Clinical Characteristics}

In patients with right temporal lobe glioma, Pearson's partial correlation analysis after controlling for the effects of age, sex, and years of education revealed that the mean ALFF value was significantly negatively correlated with visuospatial scores $(\mathrm{r}=-0.718, \mathrm{p}=0.045)$. Age, sex, and years of education were considered as covariates (Fig. 2). No significant correlation was observed between the ALFF/FC values and Digital Span Test, DSST, memory, mapping, similarity, or math test scores. In addition, no significant correlation was observed between the in- creased ALFF value and potential reorganizational time $(\mathrm{r}=0.335, \mathrm{p}=0.258)$ for all patients in this group and for patients with right temporal lobe LGG $(n=7 ; r=0.591$, $p$ $=0.204)$.

\section{Discussion}

In this study, we combined ALFF and FC analyses to evaluate possible functional compensation in patients with temporal glioma. ALFF was significantly increased in the left hippocampus and parahippocampal gyrus in patients with right temporal glioma, indicating homotopic functional plasticity of the contralesional temporal lobe. Our study provides evidence of a homotopic functional reorganization in patients with unilateral temporal glioma.

\section{Functional Plasticity of the Contralateral Homotopic Region in Patients With Right Temporal Lobe Glioma}

The brain is an entity capable of meeting various physiological and pathological demands and can effectively circumvent the expected functional deterioration of structural impairment caused by invasive tumors. ${ }^{6,14}$ The development of a lesion can affect the structural reorganization and compensation of brain function following tumor growth. ${ }^{1,7,13}$ Spatiotemporal functional compensation may explain why there is no observable clinical deficit despite tumor infiltration in the eloquent regions before and after surgery. The contralesional functional compensation seen in patients with right temporal lobe glioma is consistent with that in a previous voxel-based morphometry structural study that showed that infiltration of the unilateral insula with slow-growing glioma significantly increased the contralateral gray matter volume. Furthermore, the structural plasticity, intrinsic neural remodeling, and network reorganization in the hippocampus and parahippocampal 
Liu et al.

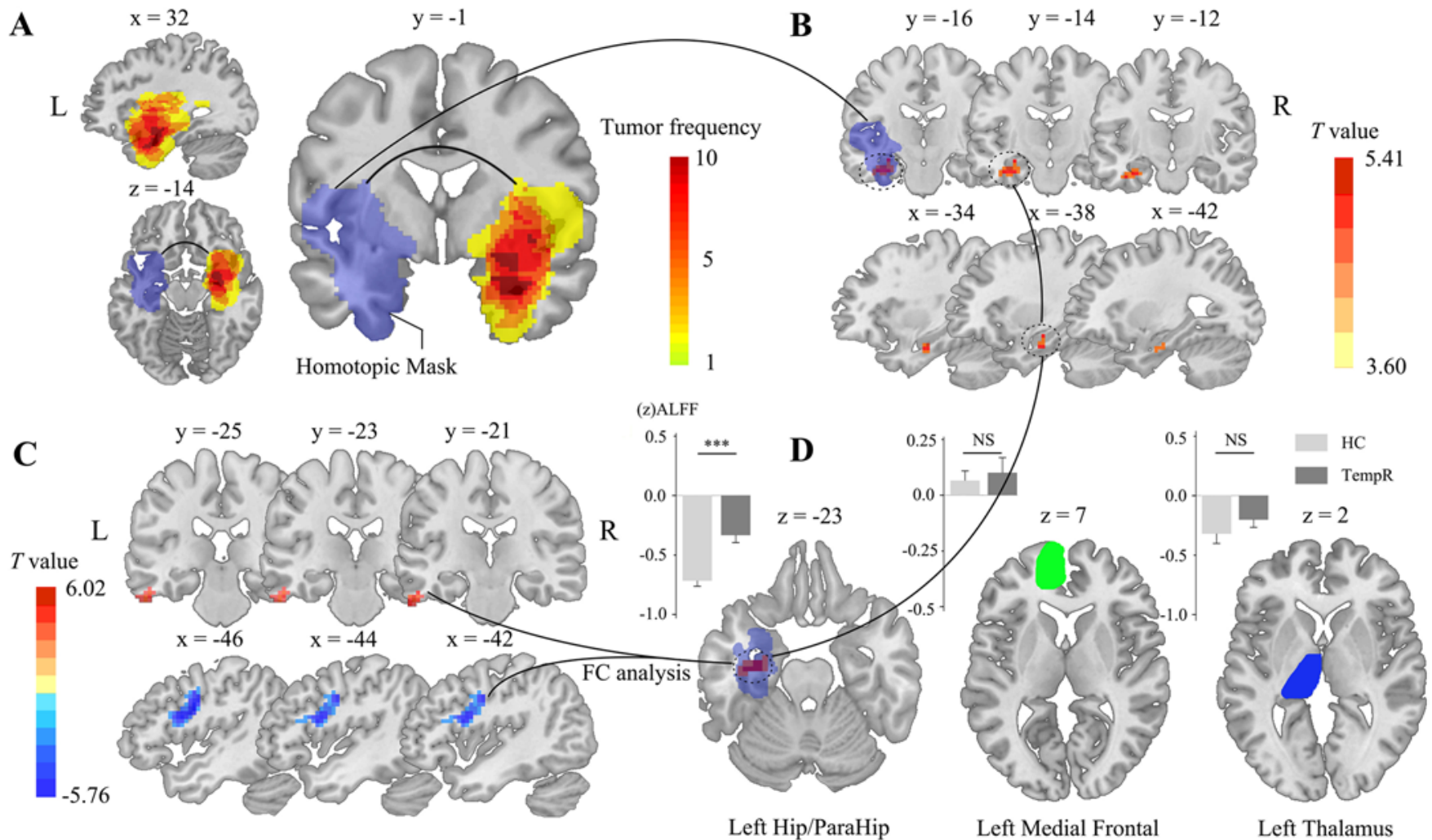

FIG. 1. A: Overlap maps of gliomas in patients with right temporal glioma. The warm colors indicate regions, the color bar represents the number of patients with a right temporal lobe glioma on each voxel, and light purple indicates the homotopic mask in contralesional left hemisphere. B: Red indicates increased ALFF within the homotopic mask in patients with right temporal glioma (permutation test, TFCE-FWE corrected $p<0.05$ ), which was selected as the ROI. C: ROI-based whole-brain FC. Red and blue indicate increased and decreased FC, respectively (voxel level $p<0.0005$ and cluster-level $p<0.05$, GRF corrected). D: ALFF in patients with right temporal glioma (TempR) compared with healthy controls (HC) in the homotopic mask (left, light blue) and 2 control ROIs (left medial frontal ROI [green] and left thalamus ROI [blue]). The histogram represents the mean standardized ALFF extracted from the region showing between-group differences in ALFF analysis and the 2 control ROls, respectively. Bars and error bars represent the mean \pm SEM. ${ }^{* * *} p<0.001$. Hip/ParaHip $=$ hippocampus and parahippocampal gyrus; NS $=$ not significant. Figure is available in color online only.

gyrus have been demonstrated in several studies. ${ }^{8,18,40}$ Sidhu et al. also detected increased left anterior hippocampal activation in patients with right temporal lobe epilepsy after anterior temporal lobe resection. ${ }^{33}$ Consistent with this, another study showed that patients with unilateral poststroke aphasia have overactive ALFF in the contralesional mesial temporal hippocampus and parahippocampal gyrus and the lateral temporal cortices. ${ }^{40}$ These phenomena could be due to the fact that terminal arborizations with high plasticity properties account for maximum synaptic turnover of adult mossy fibers in the hippocampus, ${ }^{8}$ which might have contributed to a higher functional plastic potential in the contralesional region in patients with right temporal lobe glioma. In summary, these findings imply that highly functional plasticity in the contralateral homotopic hippocampus and parahippocampal gyrus might contribute to maintaining functional balance in patients with unilateral brain lesions. This observation might have clinical significance when considering the preoperative evaluation and iterative resection of gliomas from eloquent areas of the brain..$^{29}$

Interestingly, patients with left temporal lobe glioma did not exhibit a similar functional compensation in the contralesional homotopic regions (i.e., the right hippocampus and parahippocampal gyrus) as we expected. We surmise that this result, besides the small number of patients, might be due to the low proportion of patients with LGGs $(n=1)$ in this subgroup. In pathological conditions, studies have shown that contrahemispheric plasticity is more effective in progressive rather than extensive and acute injuries. ${ }^{1,14}$ Consistent with this, in our research, 7 of the 10 patients with right temporal lobe glioma had an LGG (WHO grade I or II), whereas only 1 of the 7 patients with left temporal glioma had an LGG. Our results provide support for the functional plasticity of the homotopic hippocampus and parahippocampal gyrus in patients with LGG. This is clinically significant when considering the resection of infiltrative tumors and may help in the evaluation and development of more effective treatment or rehabilitation strategies. Some technology, such as transcranial magnetic stimulation ${ }^{28}$ or deep brain stimulation, ${ }^{35}$ was previously reported as having the ability to inhibit or excite neural structures. By stimulating the contralateral brain region, we can evaluate the possible corresponding clinical conse- 
TABLE 2. Details of the regions showing a significant difference in ALFF or FC between the temporal glioma group and controls

\begin{tabular}{|c|c|c|c|c|c|c|c|}
\hline \multirow[b]{2}{*}{ TempR vs Controls } & \multirow[b]{2}{*}{ Region } & \multirow{2}{*}{$\begin{array}{l}\text { Peak MNI } \\
\text { Coordinate* }\end{array}$} & \multirow{2}{*}{$\begin{array}{c}\text { Cluster Size } \\
\text { (voxels) }\end{array}$} & \multicolumn{2}{|c|}{ Mean Strength $\dagger$} & \multirow[b]{2}{*}{ t Value } & \multirow[b]{2}{*}{ Corrected $p$ Value } \\
\hline & & & & TempR & $\mathrm{HCs}$ & & \\
\hline \multicolumn{8}{|l|}{ ALFF } \\
\hline TempR $>\mathrm{HCs}$ & Lt Hip \& ParaHip gyrus & $(-33,-15,-21)$ & 33 & $-0.33(0.19)$ & $-0.72(0.17)$ & 5.4103 & $<0.05 \ddagger$ \\
\hline \multicolumn{8}{|l|}{ Functional connectivity } \\
\hline TempR $<\mathrm{HCs}$ & Lt inferior frontal gyrus & $(-48,9,21)$ & 140 & $-0.10(0.09)$ & $0.21(0.14)$ & -5.762 & $<0.05 \S$ \\
\hline TempR $>$ HCs & Lt inferior temporal gyrus & $(-51,-12,-36)$ & 46 & $0.59(0.17)$ & $0.19(0.19)$ & 6.018 & $<0.05 \S$ \\
\hline
\end{tabular}

Hip = hippocampus; ParaHip = parahippocampal gyrus.

* Peak coordinates $(x, y, z)$ of the MNI brain atlas.

$\dagger$ Expressed as the mean z score (SD).

$\ddagger$ Corrected by TFCE-FWE correction (1000 times of permutation test).

$\S$ Corrected by GRF correction (voxel-level $p<0.0005$, cluster-level $p<0.05$ ).

quences before the first operation or speed up the recovery of certain functions after resection and cognitive rehabilitation, and before the second operation.

In addition, we supposed that correlation analysis might reveal the relationship between time and fMRI changes when we ignored grade but correlated time with the enhanced ALFF in patients with right temporal lobe glioma $(\mathrm{n}=10)$. Contrary to prediction, there was no significant correlation between the increased ALFF value and potential reorganization time in patients with right temporal lobe glioma $(\mathrm{n}=10)$, which held true for the LGG patients only $(\mathrm{n}=7)$. One possibility is that the small sample size might have concealed some potential relationship. A second possibility is that the real compensation time, rather than the time from a patient's first onset of symptoms, cannot be estimated or determined accurately. Future longitudinal studies focusing on the fMRI changes and their relationship with accurate compensational time will better delineate the neuroplasticity in patients with glioma.

\section{Alteration of ROI-Based Whole-Brain FC}

We defined the region showing significant betweengroup differences in ALFF analysis for ROI-based voxellevel FC analysis (only in nonlesional regions). We found two regions with completely opposite resting-state FC changes between patients with right temporal lobe glioma and controls. Complete interpretation of these changes in the intrinsic resting-state neural activity requires not only regional but also network-based perspectives. With focus on the hyperconnectivity in the left inferior temporal gyrus (Fig. $1 \mathrm{C}$, red cluster), our results indicate that the region with increased FC might play a similar role in this contralesional functional compensatory process. Even though some differences might exist, our observation was similar to that in a previous study, which showed that remote cerebellar regions with decreased ALFF exhibited increased FC in the contralesional region in patients with LGG ${ }^{43}$ Here, we propose 2 alternative explanations. One possible reason for this discrepancy is that we did not take peripheral edema and the possible effects of tumor infiltration into account, which is also crucial in neural plasticity. ${ }^{5,17}$ As a result, the lesion overlay map and the homotopic mask did not contain the inferior temporal gyrus region, and the compensatory overactivity in the homotopic region manifested as enhanced FC. On the other hand, this overactivity indicates a compensatory relationship between the unilateral hippocampus and parahippocampal gyrus and the inferior temporal gyrus due to the increased demands of normal processes (such as memory ability). The recruitment of homologous functional compensation-related regions in the contralesional hemisphere might contribute to shifting the balance of the endogenous activity affected by unilateral lesions during the resting state.

Compared with controls, decreased resting-state FC was observed in the contralesional inferior frontal gyrus, a region remote to the lesion or the homologous mask, in patients with right temporal lobe glioma. To the best of our knowledge, the regions with decreased FC (Brodmann areas 6,44 , and 45 ) and the region within major lesion overlap (Brodmann areas 37 and 40) in patients with right temporal lobe glioma are part of the visuospatial network..$^{31,32}$ Studies showed that unilateral glioma may cause varying degrees of alterations in intrinsic FC, including local and long-range effects on the intra- and cross-hemisphere interactions. ${ }^{2,42}$ Significantly decreased FC might therefore indicate a disruption of network interactions between the behavior-specific network (i.e., visuospatial in this study) in patients with right temporal glioma.

\section{Correlation Between Intrinsic Regional Activity and Neurocognitive Assessments}

Our findings revealed the behavioral significance of contralesional homotopic compensation in patients with right temporal lobe glioma. The hippocampus and parahippocampal gyrus are involved in the memory circuit,,${ }^{15}$ and Sidhu et al. reported that the contralateral hippocampus contributes to memory recovery in patients who underwent unilateral anterior temporal lobe resection..$^{33}$ Interestingly, the imaging-behavioral correlation within these regions was negative in our study, rather than positive as routinely seen. Indeed, when evaluating neurocognitive characteristics (Table 1), our results showed that all patients with temporal glioma (both left and right) performed worse than the controls on the memory test. However, subgroup analysis showed no significant difference in the memory scores between patients with right temporal lobe glioma and controls. The only behavioral manifestation significantly (negatively) correlated within this region was vi- 


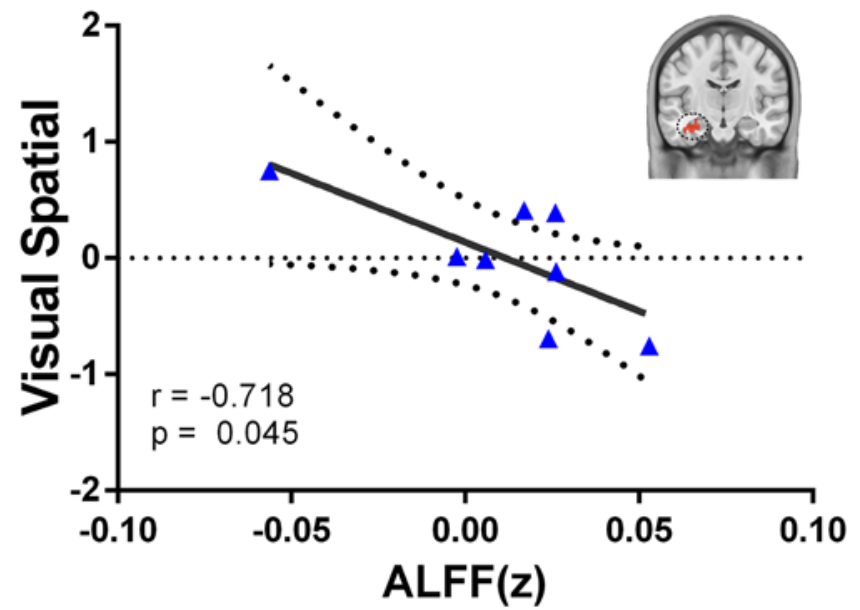

FIG. 2. Correlation between ALFF and clinical scores in patients with right temporal glioma (triangles). Two patients did not complete the visuospatial test. Standardized ALFF values in the left hippocampus and parahippocampal region were negatively correlated with visuospatial scores in patients with right temporal glioma $(r=-0.718, p=0.045)$. The solid line and dashed lines represent the best-fit line and $95 \%$ confidence interval of the Pearson partial correlation, respectively. Figure is available in color online only.

suospatial ability. Consistent with our findings, Yang et al. reported that the enhanced ALFFs in the contralesional hippocampus and parahippocampal gyrus were negatively correlated with the behavioral scores. ${ }^{40}$ We surmised that, although increased intrinsic neural activity in the homotopic region (i.e., left hippocampus and parahippocampal gyrus) might reflect-or at least tend to reflect-memoryrelated functional compensation, this compensation did not contribute to the visuospatial abilities observed in the partial correlation analysis. These findings indicated that high intrinsic regional neural activity in the contralesional hippocampus and parahippocampal gyrus was associated with the high clinical deficit caused by unilateral lesions. The damaged visuospatial function in patients with right temporal lobe glioma was the result of the damage caused by lesions in that region. Consequently, the slow progress of lesion development prolonged the time of the homotopic functional compensation, which also worsened the visuospatial abilities. Therefore, we hypothesize that increased ALFF in contralesional homotopic regions is predictive of functional reorganization in patients with right temporal lobe glioma.

\section{Limitations and Future Directions}

There are several limitations in the current study that need to be addressed. First, the small sample size could have resulted in bias and obviated statistical significance in the ALFF values between in patients with left temporal lobe glioma and controls. This also prevented an ROIbased FC analysis in these patients. It is possible therefore that the contralesional reorganizational ability differs between LGG and HGG. Second, the new 2016 WHO classification system incorporates molecular biomarkers, together with classic histological features in an integrated diagnosis. The accurate molecular subtypes of gliomas could have provided us with more information and should be adopted in future studies. Third, despite the macrostructural evidence that bilateral contralesional plasticity might be a physiological basis for functional compensation, ${ }^{1}$ we still have to realize that the subjects included in this study were all right-hand dominant. Therefore, we were unable to investigate whether a nondominant temporal glioma would lead to a greater level of plasticity in the unaffected dominant side. Further studies with larger samples of patients with left temporal glioma or left-handed patients are required to verify the role of handedness on hemispheric plasticity. Fourth, many factors can contribute to the functional remodeling detected by fMRI, such as neuronal or glial cell genesis, or even blood flow, ${ }^{25}$ which were not investigated in our study. The combination of other technologies, such as diffusion tensor imaging and electrical stimulation-based tools, may provide valuable insights into the functional, structural, and neurobiological basis of this compensatory process. Last but not least, we have to bear in mind that the clinical significance of our imaging findings could not be either absolutely summarized or excluded from the specific cognitive domains that we have evaluated in our study. More comprehensive assessments of multidomain cognitive functions are needed to explain the clinical significance of the imaging findings at the individual level during this process.

\section{Conclusions}

Our results provide evidence for a homotopic functional reorganization of the left hippocampus and parahippocampal gyrus in patients with right temporal glioma (no positive findings in patients with left temporal lobe glioma due to small sample size or small proportion of LGG patients), which may contribute to memory recovery and predict the extent of visuospatial impairment in patients with right temporal glioma. Moreover, the homotopic functional alteration might be involved in potential various network interactions during such remodeling processes. Our findings provide novel insights into brain plasticity in patients with unilateral glioma.

\section{Acknowledgments}

This study was supported by a grant from the clinical medical scientific and technologic project of National Health and Family Planning Commission of the People's Republic of China (grant no. w201308), a grant from the project of Jiangsu Provincial Medical Youth Talent (grant no. QNRC2016047), the Nanjing Commission of Health and Family Planning (grant no. H201540), a grant from the medical scientific and technologic development project of Nanjing (grant no. YKK12137 and ZKX15035), and a grant from the project of Jiangsu Provincial Medical Innovation Team (grant no. CXTDA2017050).

\section{References}

1. Almairac F, Duffau H, Herbet G: Contralesional macrostructural plasticity of the insular cortex in patients with glioma: a VBM study. Neurology 91:e1902-e1908, 2018

2. Alstott J, Breakspear M, Hagmann P, Cammoun L, Sporns O: Modeling the impact of lesions in the human brain. PLoS Comput Biol 5:e1000408, 2009

3. Chen X, Lu B, Yan CG: Reproducibility of R-fMRI metrics on the impact of different strategies for multiple comparison 
correction and sample sizes. Hum Brain Mapp 39:300-318, 2018

4. Derks J, Dirkson AR, de Witt Hamer PC, van Geest Q, Hulst $\mathrm{HE}$, Barkhof F, et al: Connectomic profile and clinical phenotype in newly diagnosed glioma patients. Neuroimage Clin 14:87-96, 2017

5. Duffau H: The huge plastic potential of adult brain and the role of connectomics: new insights provided by serial mappings in glioma surgery. Cortex 58:325-337, 2014

6. Duffau H: Lessons from brain mapping in surgery for lowgrade glioma: insights into associations between tumour and brain plasticity. Lancet Neurol 4:476-486, 2005

7. Duffau H, Capelle L, Denvil D, Sichez N, Gatignol P, Lopes $M$, et al: Functional recovery after surgical resection of low grade gliomas in eloquent brain: hypothesis of brain compensation. J Neurol Neurosurg Psychiatry 74:901-907, 2003

8. Galimberti I, Bednarek E, Donato F, Caroni P: EphA4 signaling in juveniles establishes topographic specificity of structural plasticity in the hippocampus. Neuron 65:627642, 2010

9. Gallea C, Popa T, García-Lorenzo D, Valabregue R, Legrand AP, Apartis E, et al: Orthostatic tremor: a cerebellar pathology? Brain 139:2182-2197, 2016

10. Gauthier LV, Taub E, Perkins C, Ortmann M, Mark VW, Uswatte G: Remodeling the brain: plastic structural brain changes produced by different motor therapies after stroke. Stroke 39:1520-1525, 2008

11. Greicius M: Resting-state functional connectivity in neuropsychiatric disorders. Curr Opin Neurol 21:424-430, 2008

12. Gupta L, Gupta RK, Postma AA, Sahoo P, Gupta PK, Patir $\mathrm{R}$, et al: Advanced and amplified BOLD fluctuations in highgrade gliomas. J Magn Reson Imaging 47:1616-1625, 2018

13. Heiss WD, Thiel A, Kessler J, Herholz K: Disturbance and recovery of language function: correlates in PET activation studies. Neuroimage 20 (Suppl 1):S42-S49, 2003

14. Herbet G, Maheu M, Costi E, Lafargue G, Duffau H: Mapping neuroplastic potential in brain-damaged patients. Brain 139:829-844, 2016

15. Jeffery KJ: The hippocampus: from memory, to map, to memory map. Trends Neurosci 41:64-66, 2018

16. Kinno R, Ohta S, Muragaki Y, Maruyama T, Sakai KL: Differential reorganization of three syntax-related networks induced by a left frontal glioma. Brain 137:1193-1212, 2014

17. Kristo G, Raemaekers M, Rutten GJ, de Gelder B, Ramsey NF: Inter-hemispheric language functional reorganization in low-grade glioma patients after tumour surgery. Cortex 64:235-248, 2015

18. Li H, Ji C, Zhu L, Huang P, Jiang B, Xu X, et al: Reorganization of anterior and posterior hippocampal networks associated with memory performance in mesial temporal lobe epilepsy. Clin Neurophysiol 128:830-838, 2017

19. Li Y, Tan Z, Wang J, Wang Y, Gan Y, Wen F, et al: Alterations in spontaneous brain activity and functional network reorganization following surgery in children with medically refractory epilepsy: a resting-state functional magnetic resonance imaging study. Front Neurol 8:374, 2017

20. Liu D, Hu X, Liu Y, Yang K, Xiao C, Hu J, et al: Potential intra- or cross-network functional reorganization of the triple unifying networks in patients with frontal glioma. World Neurosurg 128:e732-e743, 2019

21. Liu G, Dang C, Peng K, Xie C, Chen H, Xing S, et al: Increased spontaneous neuronal activity in structurally damaged cortex is correlated with early motor recovery in patients with subcortical infarction. Eur J Neurol 22:1540 1547,2015

22. Lopour BA, Tavassoli A, Fried I, Ringach DL: Coding of information in the phase of local field potentials within human medial temporal lobe. Neuron 79:594-606, 2013

23. Lu Y, Zhao Q, Wang Y, Zhou C: Ballroom dancing promotes neural activity in the sensorimotor system: a resting-state fMRI study. Neural Plast 2018:2024835, 2018

24. Maesawa S, Bagarinao E, Fujii M, Futamura M, Motomura $\mathrm{K}$, Watanabe $\mathrm{H}$, et al: Evaluation of resting state networks in patients with gliomas: connectivity changes in the unaffected side and its relation to cognitive function. PLoS One 10:e0118072, 2015

25. May A, Hajak G, Gänssbauer S, Steffens T, Langguth B, Kleinjung T, et al: Structural brain alterations following 5 days of intervention: dynamic aspects of neuroplasticity. Cereb Cortex 17:205-210, 2007

26. Ostrom QT, Bauchet L, Davis FG, Deltour I, Fisher JL, Langer CE, et al: The epidemiology of glioma in adults: a "state of the science" review. Neuro Oncol 16:896-913, 2014

27. Parisot S, Darlix A, Baumann C, Zouaoui S, Yordanova Y, Blonski M, et al: A probabilistic atlas of diffuse WHO grade II glioma locations in the brain. PLoS One 11:e0144200, 2016

28. Pascual-Leone A, Tarazona F, Keenan J, Tormos JM, Hamilton R, Catala MD: Transcranial magnetic stimulation and neuroplasticity. Neuropsychologia 37:207-217, 1999

29. Picart T, Herbet G, Moritz-Gasser S, Duffau H: Iterative surgical resections of diffuse glioma with awake mapping: how to deal with cortical plasticity and connectomal constraints? Neurosurgery 85:105-116, 2019

30. Raysi Dehcordi S, Mariano M, Mazza M, Galzio RJ: Cognitive deficits in patients with low and high grade gliomas. $\mathbf{J}$ Neurosurg Sci 57:259-266, 2013

31. Richiardi J, Altmann A, Milazzo AC, Chang C, Chakravarty MM, Banaschewski T, et al: Correlated gene expression supports synchronous activity in brain networks. Science 348:1241-1244, 2015

32. Shirer WR, Ryali S, Rykhlevskaia E, Menon V, Greicius MD: Decoding subject-driven cognitive states with whole-brain connectivity patterns. Cereb Cortex 22:158-165, 2012

33. Sidhu MK, Stretton J, Winston GP, McEvoy AW, Symms M, Thompson PJ, et al: Memory network plasticity after temporal lobe resection: a longitudinal functional imaging study. Brain 139:415-430, 2016

34. Smith SM, Nichols TE: Threshold-free cluster enhancement: addressing problems of smoothing, threshold dependence and localisation in cluster inference. Neuroimage 44:83-98, 2009

35. Udupa K, Bahl N, Ni Z, Gunraj C, Mazzella F, Moro E, et al: Cortical plasticity induction by pairing subthalamic nucleus deep-brain stimulation and primary motor cortical transcranial magnetic stimulation in Parkinson's Disease. J Neurosci 36:396-404, 2016

36. Volz LJ, Rehme AK, Michely J, Nettekoven C, Eickhoff SB, Fink GR, et al: Shaping early reorganization of neural networks promotes motor function after stroke. Cereb Cortex 26:2882-2894, 2016

37. Voytek B, Davis M, Yago E, Barceló F, Vogel EK, Knight RT: Dynamic neuroplasticity after human prefrontal cortex damage. Neuron 68:401-408, 2010

38. Whitcroft KL, Fischer J, Han P, Raue C, Bensafi M, Gudziol $\mathrm{V}$, et al: Structural plasticity of the primary and secondary olfactory cortices: increased gray matter volume following surgical treatment for chronic rhinosinusitis. Neuroscience 395:22-34, 2018

39. Yan CG, Wang XD, Zuo XN, Zang YF: DPABI: Data processing \& analysis for (resting-state) brain imaging. Neuroinformatics 14:339-351, 2016

40. Yang M, Li J, Li Y, Li R, Pang Y, Yao D, et al: Altered intrinsic regional activity and interregional functional connectivity in post-stroke aphasia. Sci Rep 6:24803, 2016

41. Zang Y, Jiang T, Lu Y, He Y, Tian L: Regional homogeneity approach to fMRI data analysis. Neuroimage 22:394-400, 2004 
42. Zhang H, Shi Y, Yao C, Tang W, Yao D, Zhang C, et al: Alteration of the intra- and cross-hemisphere posterior default mode network in frontal lobe glioma patients. Sci Rep 6:26972, 2016

43. Zhang N, Xia M, Qiu T, Wang X, Lin CP, Guo Q, et al: Reorganization of cerebro-cerebellar circuit in patients with left hemispheric gliomas involving language network: A combined structural and resting-state functional MRI study. Hum Brain Mapp 39:4802-4819, 2018

\section{Disclosures}

The authors report no conflict of interest concerning the materials or methods used in this study or the findings specified in this paper.

\section{Author Contributions}

Conception and design: H Liu, D Liu, Chen. Acquisition of data: D Liu, Chen, X Hu, G Hu, Y Liu, Yang, Xiao, Zou. Analysis and interpretation of data: D Liu, Chen, X Hu, Y Liu, Zou. Drafting the article: H Liu, D Liu, Chen, X Hu. Critically revising the article: H Liu, Chen, X Hu, G Hu, Y Liu, Yang, Xiao, Zou. Reviewed submitted version of manuscript: H Liu, X Hu, G Hu, Y Liu, Yang, Xiao, Zou. Approved the final version of the manuscript on behalf of all authors: H Liu. Statistical analysis: H Liu, D Liu, G Hu. Administrative/technical/material support: H Liu. Study supervision: H Liu, Zou.

\section{Supplemental Information}

\section{Online-Only Content}

Supplemental material is available with the online version of the article.

Supplemental Data. https://thejns.org/doi/suppl/10.3171/2019. 11.JNS191982.

\section{Correspondence}

Hongyi Liu: The Affiliated Brain Hospital of Nanjing Medical University, Jiangsu, China. njnkyylhy@163.com. 